Chirurg 2013 $\cdot 84: 1073$

DOI 10.1007/s00104-013-2655-0

Online publiziert: 14. November 2013

๑) Springer-Verlag Berlin Heidelberg 2013
H. Dralle

Universitätsklinik für Allgemein-, Viszeral- und Gefäßchirurgie,

Universitätsklinikum Halle, Medizinische Fakultät, Halle (Saale)

\title{
Antibiotikaprophylaxe bei Schilddrüsenoperationen
}

\section{Originalpublikation}

De Palma M, Grillo M, Borgia G et al (2013) Antibotic prophylaxis and risk of infections in thyroid surgery: results from a national study (UEC - Italian Endocrine Surgery Units Association). Updates Surg 65:231-216

\section{Hintergrund und Fragestellung}

Die Wundinfektionsrate nach Standardeingriffen der Schilddrüse ist allgemein niedrig, sie liegt im niedrig einstelligen Bereich. Obwohl von den Fachgesellschaften eine routinemäßige Antibiotikaprophylaxe bei Schilddrüsenoperationen nicht empfohlen wird, ist der Verbreitungsgrad der Antibiotikaprophylaxe bei dieser Indikation in der klinischen Praxis nicht gering, sie betrug nach den Ergebnissen einer italienischen Multicenterstudie fast $40 \%$. Die unter 38 endokrin-chirurgischen Zentren in Italien durchgeführte Erhebungsstudie diente daher dem Ziel, herauszufinden, ob die Wundinfektionsrate nach Schilddrüsenoperationen unter Antibiotikaprophylaxe niedriger war als ohne Antibiotikaprophylaxe.

\section{Methoden}

Als potenziell wundinfektionsrelevante Parameter wurden von allen in der Zeit vom 01.01.2009 bis 31.12.2011 durchgeführten Schilddrüsenoperationen Patientenalter und -geschlecht, Antibiotikaprophylaxe, Art der Operation, Drainage, Art der Schilddrüsenerkrankung und Chirurgenerfahrung (Anzahl der Schilddrüsenoperationen pro Jahr) in die Analyse einbezogen.

\section{Ergebnisse}

Unter Antibiotikaprophylaxe erfolgten 1132 von insgesamt 2926 Schilddrüsenoperationen (38,7\%). 40\% der Operationen waren multinoduläre Knotenstrumen, 15\% Karzinome. 66\% der Chirurgen führten 100 und mehr Schilddrüsenoperationen pro Jahr durch. In 90\% war das Eingriffsausmaß eine totale Thyreoidektomie, in $10 \%$ eine Hemithyreoidektomie. Die univariate Analyse zeigte eine positive Infektkorrelation mit dem Fehlen einer Wunddrainage und keiner Antibiotikaprophylaxe. Da jedoch 10 der insgesamt 28 Wundinfektionen in einem einzigen Zentrum beobachtet wurden, das keine Antibiotikaprophylaxe einsetzte, führte die anschließend durchgeführte Regressionsanalyse zu dem Ergebnis, dass das Fehlen einer Antibiotikaprophylaxe kein unabhängiger Risikofaktor für eine Wundinfektion war. Unter Ausschluss des Zentrums mit der hohen Wundinfektionsrate $(8,8 \%)$ lag die Infektrate bei $(0,4 \%)$ bzw. $0,8 \%$ mit bzw. ohne Antibiotikaprophylaxe $(\mathrm{p}=0,118)$.

\section{Diskussion und Fazit}

Die Studie hat eine Reihe von Schwächen (nicht prospektiv-randomisiert, kein Einbezug der Operationsdauer, keine Angaben zu den Infektfolgen, keine Angabe zu den Infektursachen im Zentrum mit den höchsten Infektraten), zeigt jedoch, dass trotz der allgemein niedrigen Wundinfektionsrate diese erheblich schwanken kann.

Da postoperative Wundinfektionen nach Schilddrüsenoperationen zu gra- vierenden, in Einzelfällen bis hin zu letalen Komplikationen führen können, wäre es angebracht, zur Frage der routinemäßigen Antibiotikaprophylaxe bei Standardoperationen und erweiterten Eingriffen an der Schilddrüse (Lymphadenektomie) eine Outcomeorientierte, prospektiv-randomisierte Studie unter Einschluss der Operationsdauer und des Eingriffsausmaßes durchzuführen.

\section{Korrespondenzadresse}

Prof. Dr. Dr. h.c. H. Dralle

Universitätsklinik für Allgemein-, Viszeral- und Gefäßchirurgie, Universitätsklinikum Halle, Medizinische Fakultät,

Ernst-Grube-Str. 40, 06097 Halle (Saale) henning.dralle@uk-halle.de

Interessenkonflikt. H. Dralle gibt an, dass kein Interessenkonflikt besteht. 